\title{
Numerical Investigation of Bile Secretion and Pressure Rise in Obstructed Human Common Bile Duct
}

\author{
M. Baghaei, M. Kavian, S. Ghodsi ${ }^{\dagger}$ and S. Esmail Razavi \\ Faculty of Mechanical Engineering, University of Tabriz, Tabriz, Iran \\ †Corresponding Author Email: Sina.ghodsi444@gmail.com
}

(Received January 2, 2020; accepted July 3, 2020)

\begin{abstract}
A fully obstructed Common Bile Duct (CBD) could lead to severe implications such as jaundice, cholangitis, and pancreatitis. A 2-D CFD model with the employment of Fluid-structure Interaction (FSI) formulations is established to investigate the interactions of bile with its surroundings. Ascertaining bile secretion against a total CBD obstruction is a major interest of this study. Therefore, a function is assigned to bile secretion pattern such that the resulting intraluminal pressure complies with clinical data. To cover the variation in the parameters representing the mechanical properties of the biliary system as well as bile secretion, specific piecewise ranges are given for each of them and consequently, numerous cases are simulated. Models which after simulation lead to pressure rises in the interval of $700 \mathrm{~Pa}$ to $1300 \mathrm{~Pa}$ are picked. This interval could roughly include the actual pressure rise of a vast majority of patients. It is determined that among numerous cases, higher distention does not necessarily correlate with higher pressure increase. Furthermore, the effect of alteration in each parameter in pressure rise is determined. This model is the first numerical step towards understanding the pathogenesis of complications resulting from a fully obstructed CBD and deformation of the CBD in general.
\end{abstract}

Keywords: Fluid-structure interaction; Biofluids; ALE method; Choledocholithiasis; CBD dilation.

\section{NOMENCLATURE}

$\begin{array}{ll}\text { 2-D } & \text { two-dimensional } \\ \text { 3-D } & \text { three-dimensional } \\ \text { BD } & \text { Bile Duct } \\ \text { CBD } & \text { Common Bile Duct } \\ \text { CD } & \text { Cystic Duct } \\ \text { CFD } & \text { Computational Fluid Dynamics } \\ \text { CHD } & \text { Common Hepatic Duct } \\ \text { Em }_{m} & \text { elastic moduli of the medium } \\ \text { E }_{\mathrm{w}} & \text { elastic moduli of the bile duct wall } \\ \text { FEM } & \text { Finite Element Method } \\ \text { FSI } & \text { Fluid-Structure Interaction } \\ \text { FVM } & \text { Finite Volume Method } \\ \text { I } & \text { identity tensor } \\ \text { LHD } & \text { Left Hepatic Duct } \\ \text { p } & \text { pressure } \\ \text { Q }_{s} & \text { normal bile secretion rate of the liver } \\ \text { Q }_{1} & \text { steady rate of bile secretion after } \\ & \text { obstruction }\end{array}$

\section{INTRODUCTION}

Human biliary tract is responsible for secretion, conveyance, storing and releasing bile into digestive tract in order to aid the digestion of fats (Fig. 1.).

\begin{tabular}{|c|c|}
\hline Qinlet & hepatic bile inlet \\
\hline$Q_{\text {net }}$ & $\begin{array}{l}\text { net rate of bile flow into the common } \\
\text { bile duct obtained by the difference } \\
\text { between liver secretion and the bile } \\
\text { reflux to the gallbladder }\end{array}$ \\
\hline RHD & Right Hepatic Duct \\
\hline $\mathrm{t}$ & time \\
\hline $\mathbf{u}_{\mathrm{s}}$ & solid displacement vector \\
\hline $\mathbf{v}_{\mathrm{f}}$ & fluid velocity vector \\
\hline $\mathbf{v}_{\mathrm{m}}$ & $\begin{array}{l}\text { velocity vector of the moving ALE } \\
\text { frame }\end{array}$ \\
\hline $\mathbf{v}_{\mathrm{s}}$ & solid velocity vector \\
\hline$\beta$ & decay rate in the hepatic inlet function \\
\hline$\rho_{\mathrm{s}}$ & density of the bile \\
\hline$\rho_{\mathrm{f}}$ & density of the bile duct wall \\
\hline$\mu$ & viscosity of the bile \\
\hline$\tau$ & stress tensor \\
\hline
\end{tabular}

Liver secretes bile into small intrahepatic ducts. These ducts increase in caliber as they advance towards the hilum of the liver. The common hepatic duct (CHD) emerges from the confluence of right and left hepatic ducts (RHD and LHD) outside the 
liver. Cystic duct $(\mathrm{CD})$ - which is connected to the gallbladder - joins the CHD to form the Common bile duct (CBD). Approaching downwards, the CBD courses through the duodenal wall. Bile is continuously secreted and between meals, stored in the gallbladder. During a meal, gallbladder contracts, leading to the bile being propelled into the duodenum (Yamada et al. 2009; Feldman et al. 2015).

Several studies have investigated the fluid mechanics of the biliary system. Ooi et al. (2004) carried out a Finite Volume numerical investigation on the fluid dynamics of the bile in CD. They proposed a correlation between the CD geometry and gallstone formation. Luo et al. furthered the studies on the understanding of the mechanical behaviors of the biliary tract. Their study included bile fluid dynamics, bile rheology, gallbladder and duct tissue mechanics, mathematical and numerical modeling, and the relationship between mechanical stresses and the gallbladder pain (Luo 2007). Li et al. (2007a) proposed one-dimensional rigid and elastic wall models to estimate the pressure drop in the human biliary system. They studied the effects of elastic module of the $\mathrm{CD}$ wall, bile flow rate and viscosity on the pressure drop. They concluded that the most significant contributing factor was the diameter of the CD. Though they considered the elasticity of the $\mathrm{CD}$, the CHD and the CBD were assumed to be rigid. In another paper, Li et al. (2008b) developed a simple gallbladder model to evaluate the mechanical factors contributing to gallbladder pain. The studied factors were pressure, ejection fraction, flow resistance, shear stress and peak shear and normal stresses. It was concluded that the model could potentially be used as a clinical diagnostic method. Followed by their 2007 paper, Li et al. (2008) extended their study by taking into account the effects of a 3D-modeled geometry and nonNewtonian behavior of bile on the fluid-structure interaction in the CBD. In these models, the nonNewtonian bile flow contributed to greater pressure drop. Al-Atabi et al. (2012) modeled realistic 3D geometries of CDs using scanned resin casts from actual patients and numerically with the aid of the Finite Volume Method (FVM) investigated these models as rigid CDs. They obtained the pressure drop along the CDs. Their evaluation showed a valid estimation of the CFD results compared to clinical data. Kuchumov et al. (2014) analytically examined flow of bile as a non-Newtonian fluid in the major duodenal papilla with a calculus. The objective was to assess the pathology degree in addition to contributing to improvement in clinical treatment methods. All these studies have provided appreciable insights regarding the fluid mechanics facet of the biliary system. However, an exhaustive investigation of the relevant literature yielded no numerical studies on the result of human CBD obstruction and its implications regarding distention and pressure rise.

Gallbladder and biliary associated diseases occurred in approximately 104 million people worldwide in 2013 and they resulted in 106,000 deaths (Vos 2016). It is worth mentioning that $15 \%$ of patients with gallbladder stones also suffer from BD stones. Among all the maladies pertinent to gallstones, the most lethal is acute bacterial cholangitis. Cholangitis occurs when a stone is impacted in the BD and causes the bile to be static. Pus under pressure in the BDs leads to rapid spread of bacteria via the liver into the blood, with blood poisoning as a result. Moreover, the diagnosis of cholangitis is often difficult (especially in the critical early phase of the disease) while methods pointing to the biliary tract as the source of sepsis (a toxic condition resulting from infection) are absent (Feldman et al. 2015; Bornman et al. 2003).

In this study, a comprehensive numerical analysis of the obstructed CBD has been conducted. BD obstruction is categorized into partial and complete. According to clinical data, a fully obstructed BD totally encumbers bile and gives considerable rise to the pressure inside the duct (Huang et al. 1969; Lygidakis and Brummelkamp 1985; Williams et al. 1967). In contrast, a partially obstructed BD shows insignificant intraductal pressure rise and increase in diameter, while bile can flow through the obstruction down the sphincter of Oddi (Dodds et al. 2011). The aim of this study is to investigate and provide an insight regarding the significant effects of the complete CBD obstruction on the biliary tree and bile secretion from the liver in the early stages after the obstruction. Furthermore, this is the first numerical investigation of a fully blocked CBD that implements FSI formulations to predict the distension and pressure rise in the biliary system over time. A function replicating the behavior of the liver secreting bile into the $\mathrm{CBD}$ is also introduced, which could help in understanding the early stages of the biliary system after a complete CBD obstruction. Studying escalated intraluminal pressure and the behavior of bile secretion in the blocked BD is of pronounced importance due to its correlation with various liver diseases (Feldman et al. 2015).

\section{MATERIALS AND METHODS}

\subsection{Geometry}

The study is conducted using a two-dimensional geometry of the BD that is modeled in accordance with typical BD geometries (Feldman et al. 2015). The geometry is obtained by passing a plane through the $\mathrm{BD}$ in an orientation that includes RHD, LHD, $\mathrm{CD}, \mathrm{CBD}$, and the stone. Though there are many variations of the RHD and the LHD (Castaing 2008; Adkins et al. 2000), for simplification purposes, the geometrical properties of the pair are taken to be identical. The length and diameter of each are $25 \mathrm{~mm}$ and $2 \mathrm{~mm}$ respectively. The length of the CHD and the CBD are 30 and $70 \mathrm{~mm}$ in the order given, and their diameter is set to $6 \mathrm{~mm}$. The geometry of the $\mathrm{CD}$ and its lumen is beyond the scope of this study. Therefore, the geometry of the CD is solely restricted to a boundary which is $2 \mathrm{~mm}$ wide and represents the diameter of the $\mathrm{CD}$. The wall thickness across all the ducts is set to be $1 \mathrm{~mm}$ (Adkins et al. 2000; Saxena and Theise 2004; Dowdy et al. 1962; Schulte et al. 1990).

The complete blockage of the CBD stems from one of the following: A stone passed through the $\mathrm{CD}$ and 
impacted at the distal $\mathrm{BD}$ or a stone formed inside the CBD or a tumor formed down the CBD (Yamada et al. 2009; Feldman et al. 2015). Since these would lead to similar results, the blockage is assumed to be caused by a stone. Concerning the migration of the stone, its mean diameter is around $10 \mathrm{~mm}$ (Frossard et al. 2000).

For a more accurate simulation, the implementation of a circular solid medium with the diameter of 300 $\mathrm{mm}$ around the BD was considered. This medium serves as a model for the numerous organs adjacent to the biliary tree which restrict the swelling of the BD and affect the pressure of the bile as well. The geometry of the model used for the simulations is illustrated in Fig. 2.

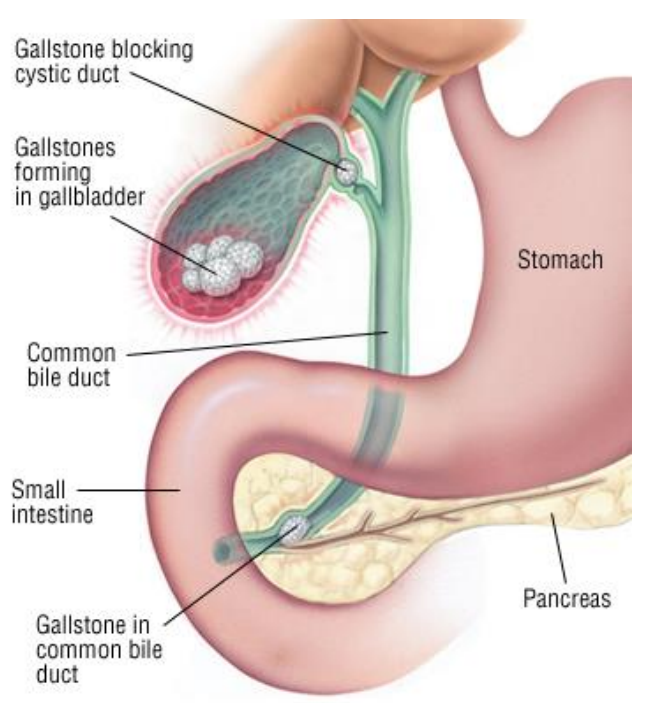

Fig. 1. Anatomy of the biliary system and different stone locations (Biliary Colic 2019).

\subsection{Bile Properties}

The flow of the bile ranges from 0.5 up to 35 in terms of the Reynolds number, thus suggesting laminar flow assumption. Although the hepatic bile shows non-Newtonian behavior in some patients, it is mostly considered to be Newtonian with the viscosity value of near water (Jüngst et al. 2001; Reinhart et al. 2010). In this study, bile viscosity is chosen to be $1 \mathrm{mPa} s$ and its density $1000 \mathrm{~kg} \mathrm{~m} \mathrm{-3}$.

\subsection{Bile Secretion}

Liver has a pressure-sensitive operating window for bile secretion. This pressure ranges from around 500 $\mathrm{Pa}$ to $1000 \mathrm{~Pa}$ over the duodenal pressure (Feldman et al. 2015; Dodds 2011; Tanaka et al. 1983). In the case of a total obstruction, relatively high pressure hinders bile secretion and consequently, bile flow into the CBD decreases drastically (Strasberg et al. 1982). This is evident in computer simulations in the sense that the increase in pressure and distension as a result of a normal liver secretion would be significantly higher than what clinical data suggests. It became apparent through simulations that the secretion should decrease significantly in a short period of time, while the pressure heightens to a certain extent. The secretion will continue to lower until it reaches a relatively stable rate. From then onwards, the pressure will continue to rise steadily until at least the next 2 days. In this time period, the relative high pressure inside the CBD will force some bile into the gallbladder. This reflux causes the gallbladder to double in volume each day for the first four days after the obstruction. The chief interactions occur in the first day and in the second day, the rate of pressure escalation follows suit (Raptopoulos et al. 1985; Kountouras et al. 1985; Duch et al. 2002). Hence, developing a function that reproduces the liver secretion behavior in the first 24 hours would be beneficial. Two identical flow inlets for the RHD and LHD in the forms of descending exponential functions are decided upon, while they seemingly represent the real reaction of the secretion to the obstruction faithfully. With respect to the mentioned points, the rate of bile secretion is defined by a function as follows:

$Q_{\text {inlet }}=\mathrm{e}^{-\beta \mathrm{t}}\left(\mathrm{Q}_{\mathrm{s}}-\mathrm{Q}_{\mathrm{l}}\right)+\mathrm{Q}_{\mathrm{l}}$

where $\mathrm{Q}_{s}$ is the normal bile secretion of the liver, $\mathrm{Q}_{1}$ is the steady rate of bile secretion after obstruction, $t$ is time in seconds and $\beta$ is the decay rate.

Liver secretes, in a normal situation, around $600 \mathrm{ml}$ up to over $1400 \mathrm{ml}$ of bile each day (Yamada et al. 2009; Feldman et al. 2015, Dodds et al. 2011). This is set as the starting point for the function $\left(\mathrm{Q}_{\mathrm{s}}\right)$. The secretion will eventually reach a steady rate $\left(Q_{1}\right)$. The rate obtained by the difference between liver secretion and the bile reflux to the gallbladder will be referred to as $\mathrm{Q}_{\text {net }}$ and governs the slope at which the pressure rises for the majority of the time period. It is assumed that, with the increase in pressure, the bile flows into the gallbladder. The gallbladder doubles in size in the first day (Raptopoulos et al. 1985). The mean volume of a normal human gallbladder is around $30 \mathrm{ml}$ (Yamada et al. 2009). Therefore, total volume refluxed to the gallbladder in a full day is $30 \mathrm{ml}$ and the uniform flow rate into the CBD is determined as $30 \mathrm{ml}$ day -1 . Different values of $\beta$ correspond to various decay times to the steady flow rate. This parameter will specially become handy for studying numerous possibilities in which the liver secretion may respond to an obstruction.

Table 1 Combinations of parameters affecting the hepatic inlet

\begin{tabular}{|c|c|c|c|c|}
\hline $\begin{array}{c}\mathrm{E}_{\mathrm{w}} \\
(\mathrm{MPa})\end{array}$ & $\begin{array}{c}\mathrm{E}_{\mathrm{m}} \\
(\mathrm{MPa})\end{array}$ & $\begin{array}{c}\mathrm{Q}_{\mathrm{s}} \\
(\mathrm{ml} / \text { day })\end{array}$ & $\begin{array}{c}\mathrm{Q}_{\text {net }} \\
(\mathrm{ml} / \text { day })\end{array}$ & $\beta$ \\
\hline 0.1 & 0.04 & 600 & 0.206 & 0.3 \\
\hline 0.88 & 0.052 & 810 & 0.279 & 0.475 \\
\hline 1.66 & 0.062 & 1020 & 0.352 & 0.65 \\
\hline 2.44 & 0.076 & 1230 & 0.424 & 0.825 \\
\hline 3.22 & 0.088 & 1440 & 0.497 & 1 \\
\hline 4 & 0.1 & & 0.570 & \\
\hline
\end{tabular}

\subsection{BD Wall}

There have been few studies on the mechanical 


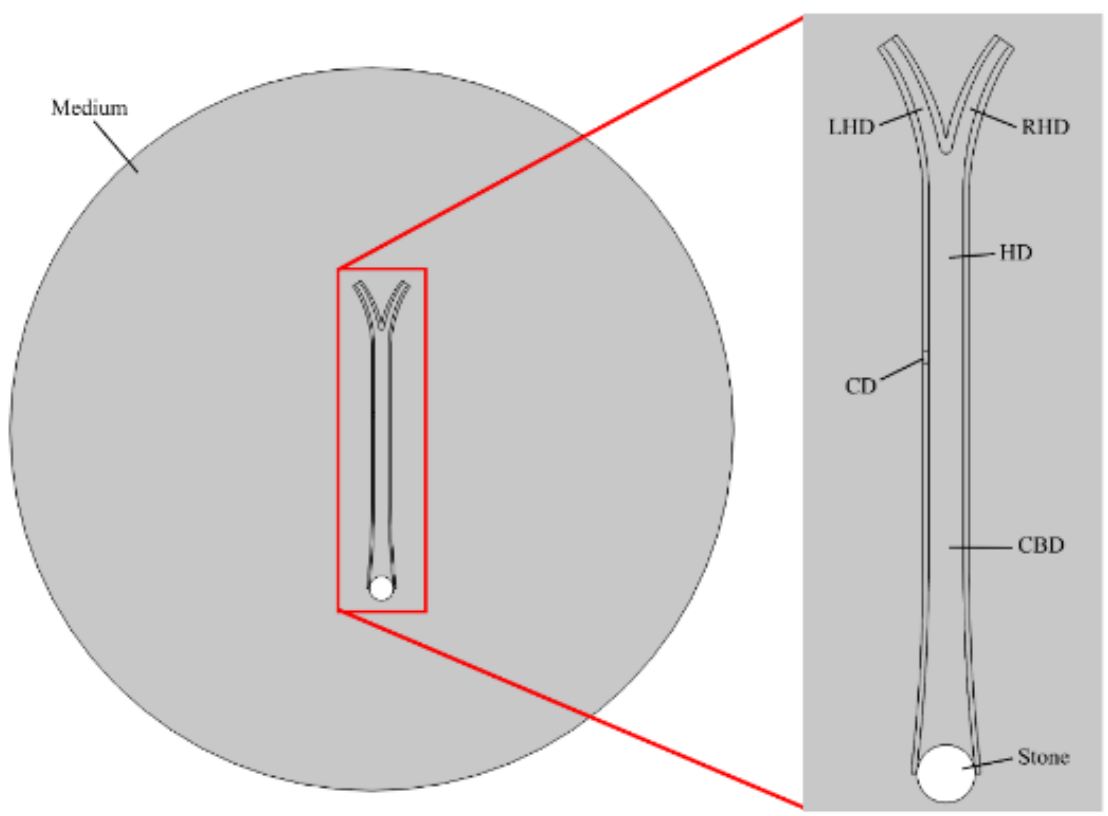

Fig. 2. Modeled investigated system comprising of the hepatic and cystic inlets, CBD, impacted stone and the medium.

behavior of the BD wall both on animals (Jian and Wang 1991; Duch et al. 2004] and humans (Girard et al. 2019) These investigations have shown that the BD wall acts in an anisotropic and non-linear manner as the stresses change. Due to lack of data, the BD wall is taken as a homogeneous isotropic linear elastic material with the density of $1150 \mathrm{~kg} \mathrm{~m}-3$ and the Poisson ratio of 0.4

\subsection{Gallbladder}

In the case of a fully obstructed CBD, the contractions of the gallbladder are neglected due to its incapability in propelling bile into CBD when subjected to high intraluminal pressures (Dodds et al. 2011).

\subsection{Combinations}

Properties of the human biliary system is patientspecific. In addition, these properties vastly vary in the clinical studies. Here, to cover adequate amount of conditions that have either direct or indirect effects on the pressure rise and bile secretion and to result in a more reliable and consummate conclusion, a method has been implemented as follows:

Five parameters including elastic moduli of the BD $\left(E_{\mathrm{w}}\right)$ and the medium $\left(E_{\mathrm{m}}\right)$, flow rates $Q_{s}$ and $Q_{n e t}$, and $\beta$, play consequential roles in pressure rise and distention. There are many similarities between human biliary tract and that of other animals ( $\mathrm{Li}$ et al. 2013). According to the stress-strain curves for the CBD and the BD wall of porcine, dog and human (Jian and Wang 1991; Duch et al. 2004; Girard et al. 2019) and the effect of aging (Sherratt 2009), the minimum and maximum amount of $\mathrm{E}_{\mathrm{w}}$ are selected to be $0.1 \mathrm{MPa}$ and $4 \mathrm{MPa}$ respectively. The maximum and minimum values for $\mathrm{E}_{\mathrm{m}}$ are based on the elastic moduli and the stress-strain curves of the adjacent organs, body fat, and muscle (Rosen et al.
2008, Sparks et al. 2015). Hence, the upper and lower limits for $\mathrm{E}_{\mathrm{m}}$ are assumed to be $0.04 \mathrm{MPa}$ and $0.1 \mathrm{MPa}$ respectively. The density of the medium is taken to be $1050 \mathrm{~kg} \mathrm{~m} \mathrm{-3}$ and the Poisson ratio of 0.4 . The lower and upper values for $\mathrm{Q}_{\text {s }}$ are supposed to be 600 and $1440 \mathrm{ml}$ day -1 respectively which could be effected by various bodily conditions (Ferland et al. 1989; Cook et al. 1950; Slater and Delaney 1971; Reyes and Kern 1979; St. George et al. 1994). Through simulations, $\mathrm{Q}_{1}$ and $\beta$ are obtained in a way to meet clinical data. Ranges of 30.206-30.57 ml day -1 and $0.3-1$ are acquired for $\mathrm{Q}_{1}$ and $\beta$ respectively. The interval of each parameter is divided into up to 6 evenly distributed values. This results in a wide but manageable number of combinations of parameters to be simulated. The total number of these combinations is 5400. According to clinical data, intraluminal pressure escalates $1000 \mathrm{~Pa}$ in the first 24 hours after complete CBD obstruction (Duch et al. 2002; Carlson et al. 1977). On this account, out of all 5400 combinations, those who yield intraluminal pressure-rises in the region of $1000 \mathrm{~Pa}$ after 24 hours are selected for further investigation.

\subsection{Initial and Boundary Conditions}

In a normal $\mathrm{CBD}$, intraluminal pressure lies between 500 and $1000 \mathrm{~Pa}$ above the duodenal pressure. Pressures over $1000 \mathrm{~Pa}$ are considered abnormal (Dodds et al. 2011). Thus, initial pressure of the CBD is assumed to be $1000 \mathrm{~Pa}$ and is set to 0 as the reference for calculation and representation of pressures after obstruction.

The boundary conditions of the edges of the LHD and RHD are fixed and as mentioned earlier, a function is set to the inlet velocity.

A constant velocity, as obtained before, is assigned to the cystic outlet to represent the steady rate of bile reflux into the gallbladder. The edges of the outlet 


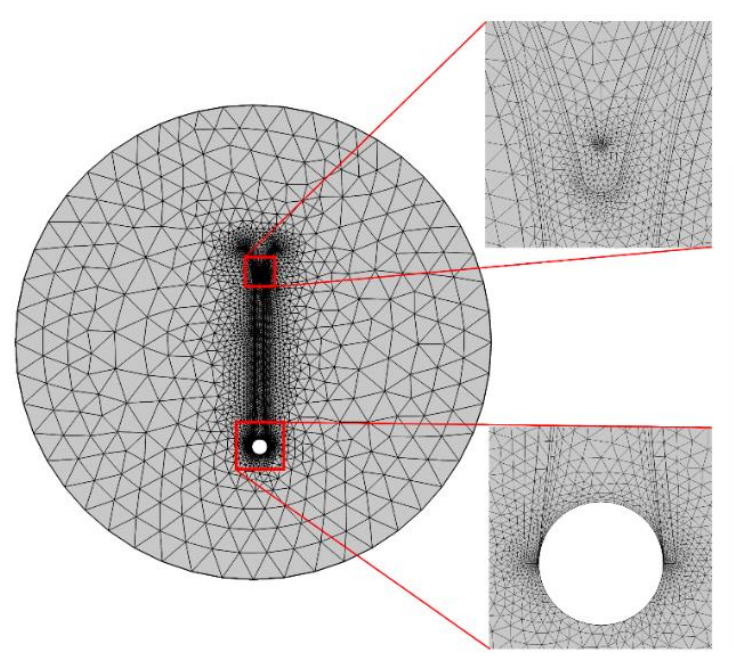

A

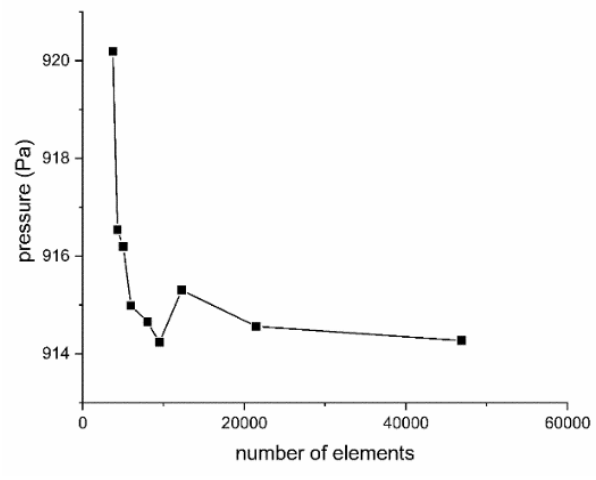

B

Fig. 3. (A) Mesh configuration of the system (B) Mesh dependence analysis.

move with the velocity of the moving boundary. The stone is assumed to be fixed. Furthermore, the outer edges of the medium circle are fixed. Fluid-structure interaction formulations are applied to all the inner walls of the RHD, LHD, CHD and the CBD.

\subsection{Numerical Simulation}

To account for the interaction between the fluid and the solid structure, Arbitrary Lagrangian-Eularian (ALE) formulation is employed. In this case, fluid flow is described using Navier-Stokes equations and a deformable domain (moving mesh) and solid mechanics is formulated using Lagrangian description and an undeformed frame. The Continuity and Navier-Stokes equations for incompressible flow are given in a vector form as follows:

$\rho_{f} \frac{\partial \mathbf{v}_{\mathrm{f}}}{\partial t}-\nabla \cdot\left[-p \boldsymbol{I}+\mu\left(\nabla \mathbf{v}_{\mathrm{f}}+\left(\nabla \mathbf{v}_{\mathrm{f}}\right)^{T}\right)\right]+$

$\rho_{f}\left(\left(\mathbf{v}_{\mathrm{f}}-\mathbf{v}_{m}\right) . \nabla\right) \mathbf{v}_{\mathrm{f}}=0$

where $\rho_{f}$ is the fluid density, $\mathbf{v}_{\mathrm{f}}$ the vector of fluid velocity, $\mathbf{v}_{m}$ the velocity vector of the moving ALE frame, p the fluid pressure, and $\boldsymbol{I}$ the identity tensor. The equation of motion of the solid structure in the Lagrangian form reads:

$\rho_{s} \frac{\partial^{2} \mathbf{u}_{s}}{\partial t^{2}}=\nabla . \boldsymbol{\tau}$

where $\rho_{s}$ is the solid density, $\mathbf{u}_{s}$ the vector of structural displacements, and $\boldsymbol{\tau}$ the first PoilaKirchhoff stress tensor.

Also, both the fluid and solid structure have the same velocity at the boundary. Thus:

$\frac{\partial \mathbf{u}_{\mathrm{s}}}{\partial t}=\mathbf{v}_{\mathrm{f}}$

The CFD simulations are carried out utilizing the Finite Element Method (FEM) and Backward Differentiation Formula (BDF) for time stepping. To solve the multiphysics model, a fully coupled solver is applied. Also, second order schemes are employed for discretization of both pressure and velocity fields.

\subsection{Mesh}

Fig. 3A. depicts the meshing of the $\mathrm{BD}$ and the surrounding medium. Total number of mesh elements is 8010 and comprises of 7356 triangular and 654 quadrilateral elements. Independence of the results to the mesh is obtained (Fig. 3B.) by comparing the pressure of a point inside the duct calculated using different grades of element size.

\section{RESULTS}

For visualization and determining the effect of stone diameter, investigating mesh independence and comparing normal, partially and fully obstructed CBDs, a conservative sample combination of the parameters is chosen whose values are presented in Table 2 .

Table 2 Chosen parameters for visualization

\begin{tabular}{|c|c|c|c|c|}
\hline $\begin{array}{c}\mathrm{E}_{W} \\
(\mathrm{MPa})\end{array}$ & $\begin{array}{c}\mathrm{E}_{\mathrm{M}} \\
(\mathrm{MPa})\end{array}$ & $\mathrm{Q}_{\mathrm{s}}(\mathrm{ml} /$ day $)$ & $\begin{array}{c}\mathrm{Q}_{\text {net }} \\
(\mathrm{ml} / \text { day })\end{array}$ & $\beta$ \\
0.88 & 0.04 & 1020 & 0.57 & 0.3 \\
\hline
\end{tabular}

\subsection{Stone Diameter}

The average impacted CBD stone diameter is around $10 \mathrm{~mm}$ (Frossard et al. 2000). A variation of $4.86 \mathrm{~Pa}$, equaling to $0.53 \%$ of the total pressure rise, occurred as a result of altering the stone diameter between 8 $\mathrm{mm}$ and $16 \mathrm{~mm}$. Therefore, a fixed diameter of 10 $\mathrm{mm}$ is chosen for all the different combinations of the study.

\subsection{Normal and Partially Obstructed CBD}

In a normal CBD (Fig. 4A.) the pressure gradient along the $\mathrm{CBD}$ is less than $1 \mathrm{~Pa}$. Running simulations for a $95 \%$ obstructed CBD (Fig. 4B.) resulted in a 
quick pressure rise of $75 \mathrm{~Pa}$ after which the system reached a steady state. A negligible diameter increase is also apparent. It can be inferred that up to at least $95 \%$ of obstruction, the system goes under very subtle changes in comparison to a fully obstructed CBD.

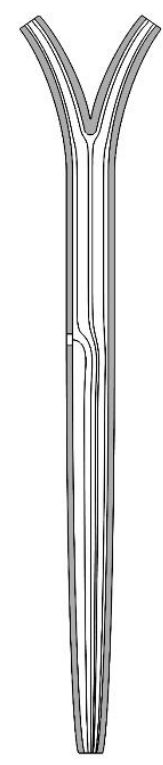

(A)

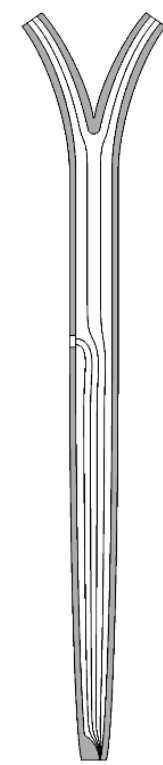

(B)
Fig. 4. Stationary streamlines visualizing the flow from the liver and the gallbladder in (A) a normal CBD and (B) a partially obstructed CBD.

\subsection{Analysis of Results Obtained by Combinations}

After choosing the combinations that result in a final pressure of between $700 \mathrm{~Pa}$ and $1300 \mathrm{~Pa}$ and thoroughly analyzing the data, a few patterns are recognized. For better showcasing the patterns, combinations are divided into 3 intervals, which include data with resulting final pressure of 700 to $900 \mathrm{~Pa}, 900$ to $1100 \mathrm{~Pa}$ and 1100 to $1300 \mathrm{~Pa}$. The data from each interval are sorted by all the parameters accordingly and the frequency of each parameter among the available combinations in their respective interval is recorded. This value is then divided by the total number of combinations of the interval to give the relative frequency.

The manifestation of each parameter is more uniform across its range in the middle batch of the data $(900 \mathrm{~Pa}<\mathrm{p}<1100 \mathrm{~Pa})$. However, specific regions of the range seem to play a major role in the combinations leading to pressures higher than $1100 \mathrm{~Pa}$ and lower than $900 \mathrm{~Pa}$. Moreover, the relative frequency of the first interval follows transversely the behavior of the third interval. This is evident in all the parameters. Figure 5A. shows the pattern concerning the relative frequency of $\mathrm{E}_{\mathrm{m}}$ for the first and third intervals as they tend to reach an extremum in the middle of the range. The same can be said for $\mathrm{E}_{\mathrm{w}}$ and $\mathrm{Q}_{\text {net }}$ (Fig. 5B. and Fig. 5C.). A disparate pattern of consistent rise and fall is plane in the $\beta$ and $Q_{s}$ relative frequencies. (Fig. 5D. and Fig. 5E.).

The results suggest that to obtain pressures outside the 900-1100 $\mathrm{Pa}$ range, the variety of the combinations are relatively limited. $38 \%$ of the data are in the 700-900 $\mathrm{Pa}$ range. This amount is $43 \%$ and $19 \%$ for $900-1100 \mathrm{~Pa}$ and $1100-1300 \mathrm{~Pa}$ ranges respectively. The limitation of combinations is also apparent in the elastic moduli and $\mathrm{Q}_{\text {net, }}$ as the major determining parameters, being more relatively frequent in certain values rather than their whole range. The opposite is exhibited in data batch of 900-1100 Pa. This indicates that a greater group of people with various biliary system specifications, as a consequence of their age, gender and circumstances, reach a similar state in biliary system pressure and a less pronounced diversity of people reach pressures outside this range. This corresponds with the clinical data which show an increase of around $1000 \mathrm{~Pa}$ in the first day after a complete CBD obstruction. A careful investigation of the $\beta$ and $Q_{s}$ relative frequencies suggest that in the case of high pressures, $\beta$ alters the hepatic function in a way that the liver reacts more reluctantly and the secretion descends relatively slow. The relative frequency of $\mathrm{Q}_{\mathrm{s}}$ also indicates that cases with higher normal liver secretion rate tend to suffer more from higher pressure levels in the case of a complete obstruction. The opposite can be inferred from the pressure levels lower than $900 \mathrm{~Pa}$. In the case of the pressures in the range of 900-1100 $\mathrm{Pa}$, the whole ranges of $\beta$ and Qs have a uniform contribution in combinations which authenticates the former deduction about diversity.

The corresponding CBD diameter for each case is plotted. Figure 6. demonstrates the observation that various factors contribute to the final distension of the CBD in the sense that a more distended CBD does not necessarily resemble higher intraluminal pressure.

The results of different combinations when scattered on a pressure-diameter plot form lines with different slopes that when forecasted backwards, cross the original state of the CBD $(6 \mathrm{~mm}$ of diameter and $0 \mathrm{~Pa}$ of pressure rise). The upper and lower limits of these lines (largest and smallest slopes) in conjunction with the lowest and highest pressure and distension limits, form an area. This area can potentially be interpreted as the area in which all cases of the CBD complete obstructions could fit into. In addition, even though the combinations are selected owing to their pressures, it is palpable from the data that the diameter range also adheres to the clinical data (Raptopoulos et al. 1985; Shawker 1981).

\subsection{Effect of time}

The result of three combinations are illustrated in Fig. 7. to demonstrate the state of pressure and diameter change as time advances. Figure 7A. exemplifies the hepatic inlet function that leads to these pressure levels and distensions. 


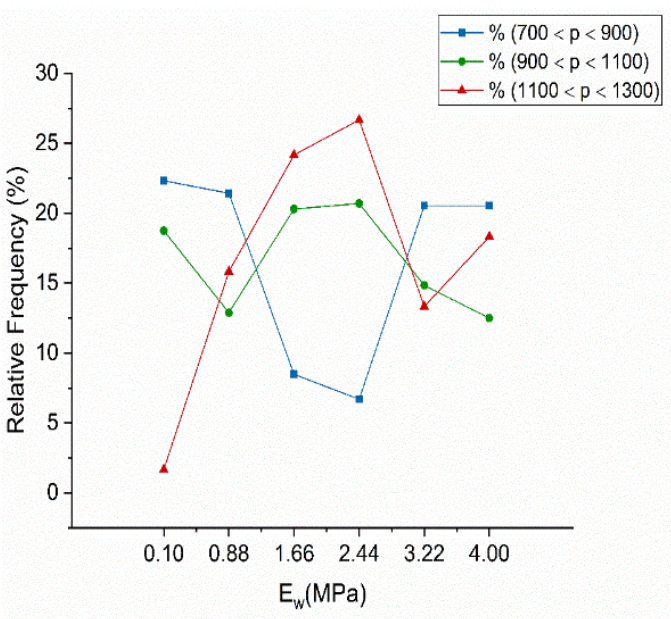

(A)

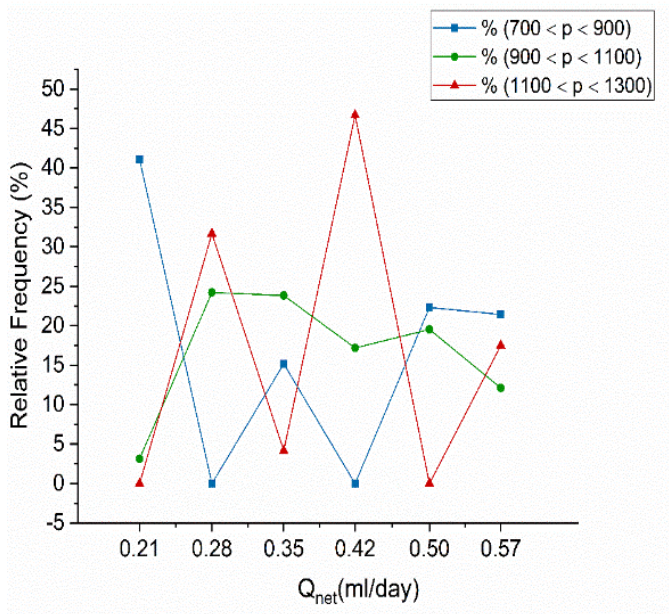

(C)

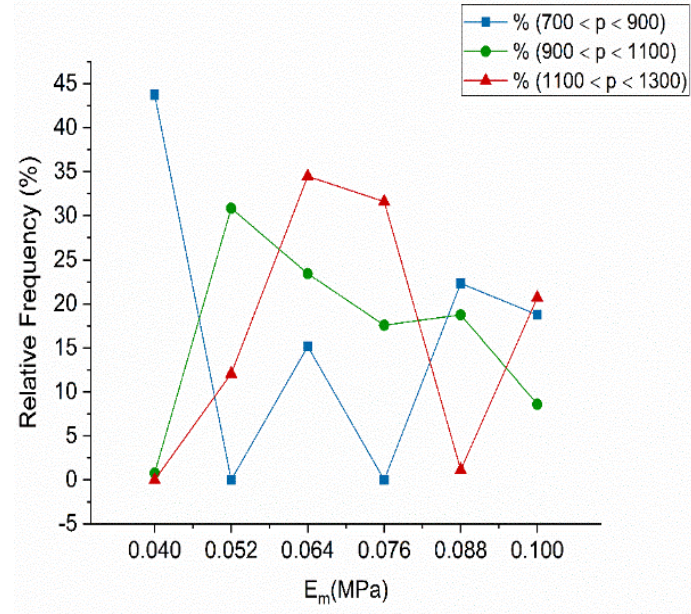

(B)

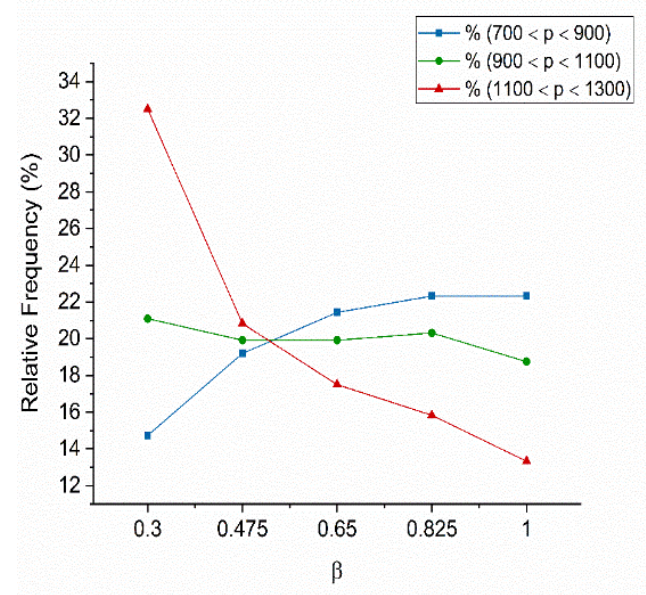

(D)

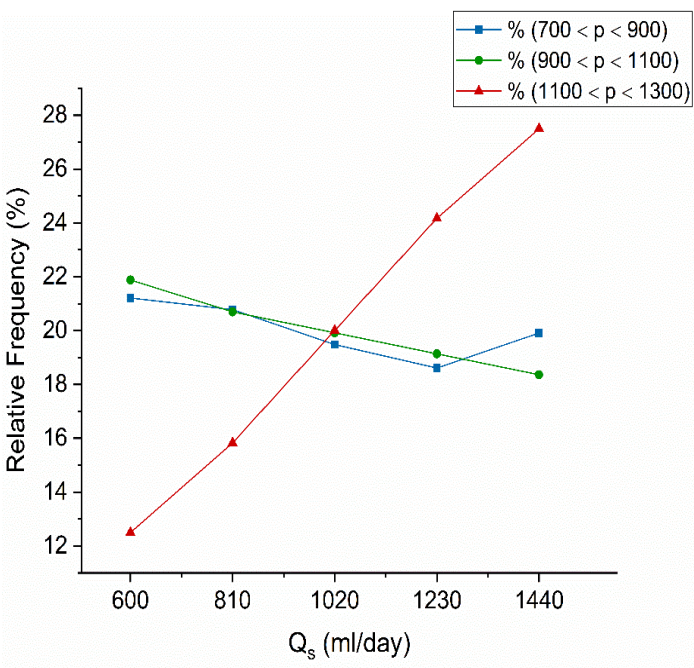

(E)

Fig. 5. Relative frequencies of elastic moduli of (A) BD wall (B) medium and consequential parameters in the defined hepatic secretion function (C) net overall inflow (D) $\beta$ (E) daily secretion of hepatic bile. 
M. Baghaei et al. / JAFM, Vol. 14, No. 1, pp. 275-286, 2021.

Table 3 Average values of the properties of two groups of cases sorted by their resulting CBD dilation

\begin{tabular}{|c|c|c|c|c|c|}
\hline & Diameter $(\mathrm{mm})$ & Pressure $(\mathrm{Pa})$ & $\mathrm{E}_{\mathrm{w}}(\mathrm{MPa})$ & $\mathrm{E}_{\mathrm{m}}(\mathrm{MPa})$ & $\mathrm{Q}_{\mathrm{s}}(\mathrm{ml} / \mathrm{day})$ \\
\hline Group I & 7.43 & 792.4 & 3.61 & 0.094 & 964.7 \\
\hline Group II & 8.76 & 997 & 1.82 & 0.065 & 1028 \\
\hline
\end{tabular}

These combinations are chosen in a way to best portray the effects of parameters $\beta$ and $Q_{\text {net. }}$ Therefore, parameters $\mathrm{E}_{\mathrm{w}}, \mathrm{E}_{\mathrm{m}}$, and $\mathrm{Q}_{\mathrm{s}}$ are kept unaltered. The effect of altering $\mathrm{Q}_{\mathrm{s}}$ would be the change in the starting point of the hepatic inlet function and variation of $E_{w}$ and $E_{m}$ would influence the resulting pressure and distension of the CBD rather than directly modifying the inlet function. Figure 7A. demonstrates that, while $\beta$ has a pronounced effect in the first phase of the pressure change, $Q_{\text {net }}$ dictates the rate at which the pressure keeps on building for the remainder of the time.

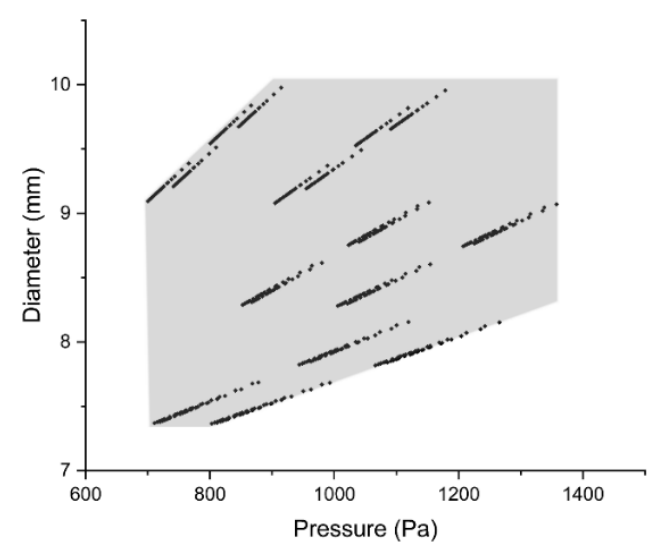

Fig. 6. Pressure-distension scatter depicting cases with different characteristics of the biliary system. Shaded area demarcates potential ranges for pressure and distension.

Figure 8. represents the velocity magnitude contours in different times and streamlines of the dilated CBD after 24 hours. A small amount of bile flows down to $\mathrm{CBD}$ and this is the reason behind the slight slope of the line after the reduction of liver secretion.

\subsection{Correlations to Clinical Conditions}

To assess the correlation of pressure and bile secretion variations with related disease such as cholangitis and jaundice, it is best to look into the upshots of the conditions; mainly distension, clinical symptoms after obstruction, etc. Since dilation of the CBD is closely related to the mechanical properties of the biliary system as well as the activity level of the liver, some relations to the aforementioned diseases can be extracted through analyzing the effects these properties and parameters have on the dilation of the CBD.
One of the challenges of cholangitis is its diagnosis especially in the early stages. Although most of the patients with acute cholangitis experience CBD dilation, it has been shown that up to $30 \%$ of the cases might not undergo CBD dilation (Balthazar et al. 1993; Bader et al. 2001; Kim et al. 2009; Hong et al. 2012). Since the main means of diagnosis is using various imaging techniques, the absence of dilation can introduce even more difficulty for the diagnosis. There have been some studies on the clinical differences between the patients with and without CBD dilation. The distinguishable physical factor among them was the small diameter change after obstruction. It has been shown that patients with severe cholangitis experience more CBD dilation and thus longer treatment time in the hospital (Hong et al. 2012). The smaller CBD dilation often means that other clinical factors such as bilirubin and white blood cell count in these patients are lower (Kiriyama et al. 2018). Though these studies have investigated the differences of clinical symptoms when the magnitude of dilation is considered, no specific reason behind this variance of diameter among the patients with cholangitis - other than small age variations (Hong et al. 2012; Tomizawa et al. 2017) - has been suggested.

Although most cases in this work fall into the middle range pressure rise, some percentage of the simulations follow the trend that is apparent in the clinical studies; around 13\% of cases (Group I) underwent dilations smaller than $1.5 \mathrm{~mm}$ in the first day. The mean pressure rise in this Group was calculated to be $792.4 \mathrm{~Pa}$. This value indicates that the cases with less severe CBD dilation (mainly categorized as Grade I) have lower pressure rise in the first stages of cholangitis. The reason can be found in the mean values of $E_{w}$ and $E_{m}$. These values are $3.61 \mathrm{MPa}$ and $0.094 \mathrm{MPa}$ respectively. The mean wall and medium elasticities are just shy of the highest values assigned to them suggesting stiffer CBD wall and its soundings - mostly attributed to younger patients, can mitigate the dilation and since it is shown that smaller dilations tend to have lower pressure rise, the lower bilirubin levels in these patients suggested by clinical data, can be related to the lower intraluminal pressures of these cases.

The other section of the data (Group II) with dilations over $1.5 \mathrm{~mm}$ has a higher mean pressure rise value of around $997 \mathrm{~Pa}$. It can be observed that higher intraluminal pressures are resulting in more CBD dilation thus having more severe conditions when clinical factors are considered. The average values of studied parameters are available in Table 3. 
M. Baghaei et al. / JAFM, Vol. 14, No. 1, pp. 275-286, 2021.

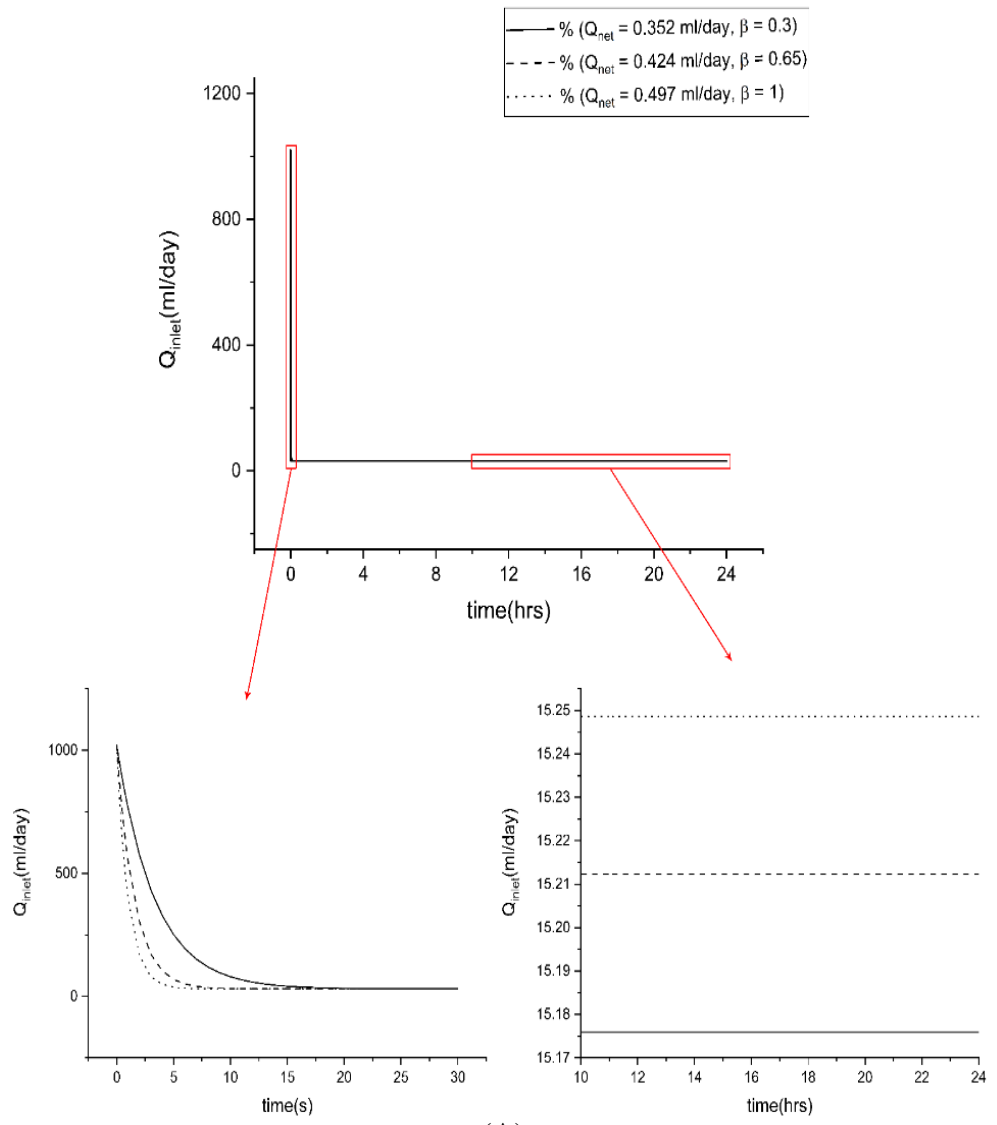

(A)

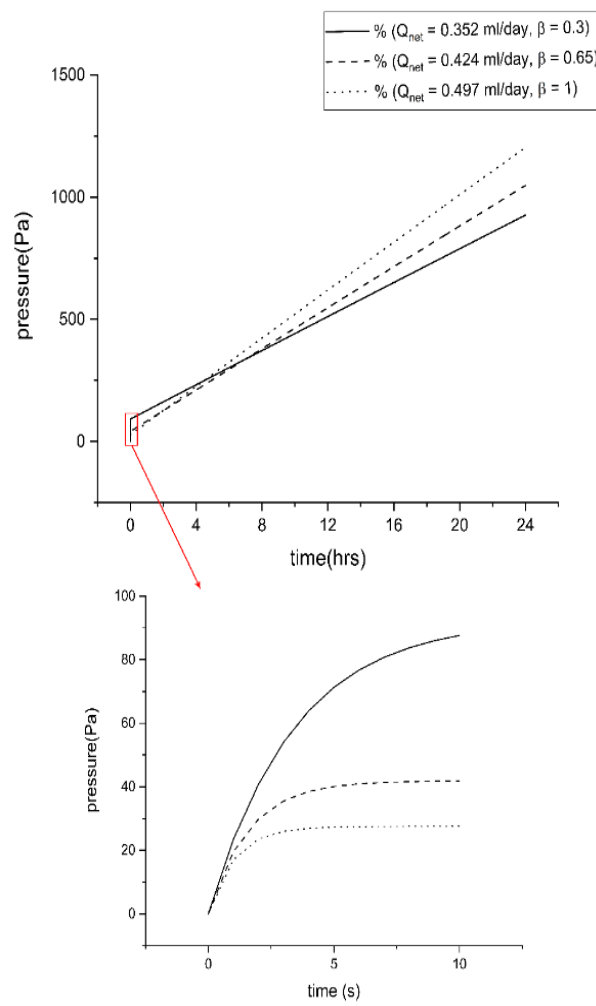

(B)

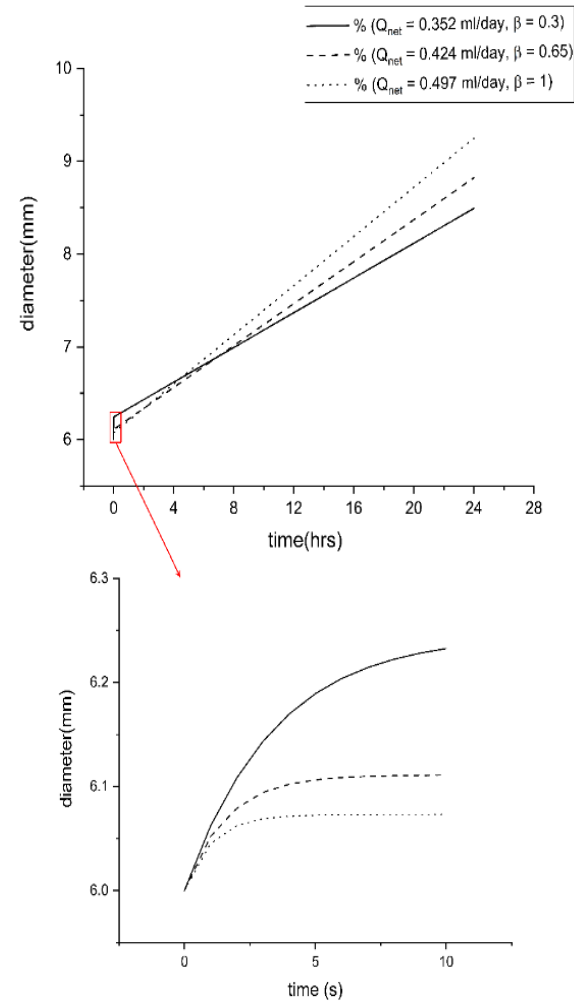

(C)

Fig. 7. (A) Hepatic function verses time, specifying the role of $\beta$ and Qnet. Varying aspects of an obstructed CBD as the time advances: $(B)$ pressure and $(C)$ diameter. 


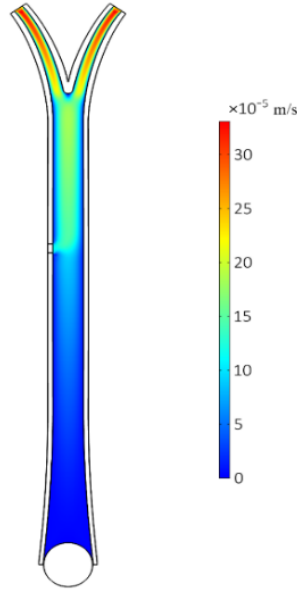

(A)

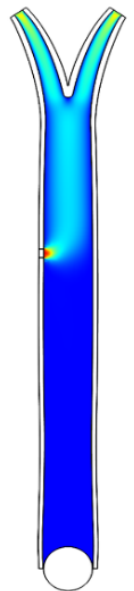

(B)
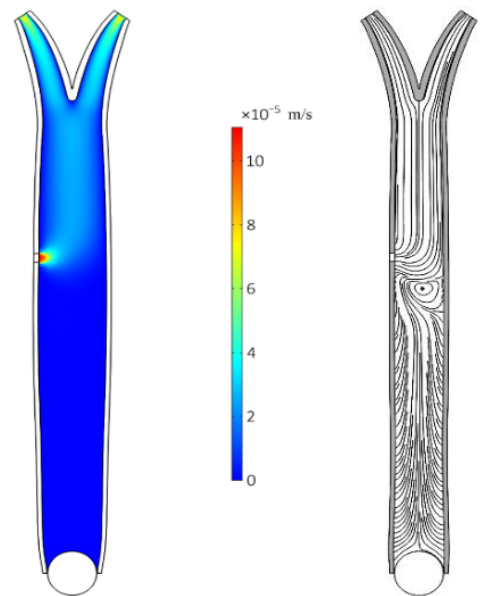

(C)

(D)

Fig. 8. Effect of time on diameter and contours of velocity magnitude at: (A) $t=10 \mathrm{~s}(\mathrm{~B}) t=24$ hours (C) $t=48$ hours and streamlines of the flow after 24 hours (D).

It is observed that generally in the cases with lower elasticity moduli of the CBD wall and its surroundings the resulting pressure and stress is not as high. With that in mind, it is evident form Table 3 that the pressure rise in group II is higher than that of group I, even though its average elastic moduli is relatively small. This can be explained by their higher average normal daily bile secretion and probably the slower reaction of their biliary system to a total obstruction that leads to the situation with higher intraluminal pressure levels and more CBD dilation.

\section{CONCLUSION}

The behavior of the biliary system in the presence of an obstruction is of great concern. It was evident that no significant pressure change happens in the case of a partially obstructed CBD. This study was targeted at getting an insight and understanding of the liver reaction, bile flow, and biliary system pressure during a complete CBD obstruction. It was decided upon that an exponential function reflects the bile secretion pattern of the liver accurately. Parameters for the liver secretion function were obtained and their ranges were determined such that different scenarios would be simulated. This function in conjunction with the elastic moduli of the BD wall and the medium play chief roles in pressure increase of the biliary system.

It was observed that the elastic modulus of the medium has a great impact on the pressure rise while simultaneously restricting the deformation of the biliary system. Furthermore, a biliary system with higher elastic moduli for its duct walls and its surroundings is not accompanied by higher liver secretion rates. This could indicate that the mechanical properties of the biliary tree determine the functioning level of the liver. While aging with the consequence of less stiffness in duct walls, could lead to higher pressure levels in the case of a complete obstruction, other conditions such as pregnancy, toxins, gender, obesity, dietary habits, etc. can either intensify or attenuate the pressure due to their respective effects on the normal rate of bile secretion. The presented function with corresponding values for its parameters could possibly cover these varieties in liver performance.

It was noticed that most cases with vastly different bile secretion levels and elastic moduli of their biliary systems do reach similar pressure levels and CBD distensions. Even though in a single case, the pressure level and distension are correlated, by comparison of different cases, it can be proven that a case with higher distension does not necessarily translate into a case with higher CBD pressure levels.

The results of the simulations were divided into two groups concerning their dilations. These groups were compared to each other and the mechanical reasons behind the variations of dilations were discussed. It was concluded that severe cases of cholangitis with higher pressure rise and more bacterial infection happen when the overall stiffness of the biliary system is lower and the liver activity is on higher levels.

The studied model is the first numerical step regarding the pathogenesis of severe consequences of a fully obstructed CBD such as cholangitis and deformation of CBD in general. While in clinical scenarios, due to certain limitations, the pressure across the whole time-interval after a complete obstruction cannot be measured, it is hoped that this study would give a reliable image of how the CBD pressure and distension reach a certain point after an obstruction.

\section{ACKNOWLEDGEMENT}

We thank Seyed Mohammad Baghaei, MD, for providing support in the clinical and medical aspects of this paper. 


\section{REFERENCES}

Adkins, R. B., W. C. Chapman and V. S. Reddy (2000). Embryology, anatomy, and surgical applications of the extrahepatic biliary system. Surgical Clinics of North America volume 80 (1), 363-379.

Al-Atabi, M., R. C. Ooi, X. Y. Luo, S. B. Chin and N. C. Bird (2012). Computational analysis of the flow of bile in human cystic duct. Medical Engineering and Physics 34 (8), 1177-1183.

Bader, T. R., L. Braga, K. L. Beavers and R. C. Semelka (2001). MR imaging findings of infectious cholangitis. Magnetic Resonance Imaging 19 (6), 781-788.

Balthazar, E. J., B. A. Birnbaum and M. Naidich (1993). Acute cholangitis: Ct evaluation. Journal of Computer Assisted Tomography 17(2), 283-289.

Biliary Colic (2019), https://www.health. harvard.edu/a_to_z/biliary-colic-a-to-z

Bornman, P. C., J. I. van Beljon and J. E. J. Krige (2003). Management of cholangitis. Journal of Hepato-Biliary-Pancreatic Surgery 10(6), 406414.

Carlson, E., C. F. Zukoski, J. Campbell and M. Chvapil (1977). Morphologic, biophysical, and biochemical consequences of ligation of the common biliary duct in the dog. American Journal of Pathology 86(2), 301-320.

Castaing, D. (2008). Surgical anatomy of the biliary tract. $H P B$ 10(2), 72-76.

Cook, D. L., D. A. Beach, R. G. Bianchi, W. E. Hambourger and D. M. Green (1950). Factors influencing bile flow in the dog and rat. The American Journal of Physiology 163(3), 688694.

Dodds, W. J., W. J. Hogan and J. E. Geenen (2011). Motility of the biliary system. Comprehensive Physiology Supplement 16. Handbook of Physiology, The Gastrointestinal System, Motility and Circulation, 1055-1101.

Dowdy, G. S., G. W. Waldron and W. G. Brown (1962). Surgical Anatomy of the Pancreatobiliary Ductal System: Observations. Archives of Surgery. 84(2), 229-246.

Duch, B. U., H. Andersen and H. Gregersen (2004). Mechanical properties of the porcine bile duct wall. BioMedical Engineering Online 3(23), 18.

Duch, B. U., H. L. Andersen, J. Smith, G. S. Kassab and H. Gregersen (2002). Structural and mechanical remodelling of the common bile duct after obstruction. Neurogastroenterology and Motility 14(2), 111-122.

Feldman, M., L. S. Friedman and L. J. Brandt (2015). Sleisenger and Fordtran's Gastrointestinal and Liver Disease- 2 Volume Set, 10th Edition. Published by: SAUNDERS ELSEVIERPhiladelphia, United States
Ferland, G., B. Tuchweber, A. Perea and I. M. Yousef (1989). Effect of aging and dietary restriction on bile acid metabolism in rats. Lipids 24(10), 842-848.

Frossard, J. L., A. Hadengue, G. Amouyal, A. Choury, O. Marty, E. Giostra, , F. Sivignon, L. Sosa, P. Amouyal (2000). Choledocholithiasis: A prospective study of spontaneous common bile duct stone migration. Gastrointestinal Endoscopy 51(2), 175-179.

Girard, E., G. Chagnon, E. Gremen, M. Calvez, C. Masri, J. Boutonnat, B. Trilling, B. Nottelet (2019). Biomechanical behaviour of human bile duct wall and impact of cadaveric preservation processes. Journal of the Mechanical Behavior of Biomedical Materials 98(2019), 291-300.

Hong, M. J., S. W. Kim, H. C. Kim and D. M. Yang (2012). Comparison of the clinical characteristics and imaging findings of acute cholangitis with and without biliary dilatation. British Journal of Radiology 85(1020), e1219e1225.

Huang, T., J. A. Bass and R. D. Williams (1969). The Significance of Biliary Pressure in Cholangitis. Archives of Surgery 98(5), 629-632.

Jian, C. and Wang, G. (1991). Biomechanical study of the bile duct system outside the liver. BioMedical Materials and Engineering 1(2), 105113.

Jüngst, D., A. Niemeyer, I. Müller, B. Zündt, G. Meyer, M. Wilhelmi and R. Del Pozo (2001). Mucin and phospholipids determine viscosity of gallbladder bile in patients with gallstones. World Journal of Gastroenterology 7(2), 203207.

Kim, S. W., H. C. Shin and I. Y. Kim (2009). Transient arterial enhancement of the hepatic parenchyma in patients with acute cholangitis. Journal of Computer Assisted Tomography 33(3), 398-404.

Kiriyama, S., K. Kozaka, T. Takada, S. M. Strasberg, H. A. Pitt, T. Gabata, J. Hata, K. H. Liau, F. Miura, A. Horiguchi, K. H. Liu, C. H. Su, K. Wada, P. Jagannath, T. Itoi, D. J. Gouma, Y. Mori, S. Mukai, M. E. Giménez, , ... M. Yamamoto (2018). Tokyo Guidelines 2018: diagnostic criteria and severity grading of acute cholangitis (with videos). Journal of HepatoBiliary-Pancreatic Sciences 25(1), 17-30.

Kountouras, J., P. J. Scheuer and B. H. Billing (1985). Effect of prolonged bile duct obstruction in the rat on hepatic transport of bilirubin. Clinical Science.

Kuchumov, A. G., V. Gilev, V. Popov, V. Samartsev and V. Gavrilov (2014). Non-newtonian flow of pathological bile in the biliary system: Experimental investigation and CFD simulations. Korea Australia Rheology Journal 26(1), 81-90.

Li, W. C., H. M. Zhang, J. Li, R. K. Dong, B. C. Yao, X. J. He, H.Q. Wang, J. Song(2013). 
M. Baghaei et al. / JAFM, Vol. 14, No. 1, pp. 275-286, 2021.

Comparison of biomechanical properties of bile duct between pigs and humans for liver xenotransplant. Transplantation Proceedings 45(2), 741-747.

Li, W. G., X. Y. Luo, A. G. Johnson, N. A. Hill, N. Bird and S. B. Chin (2007). One-dimensional models of the human biliary system. Journal of Biomechanical Engineering 129(2), 164-173.

Li, W. G., X. Y. Luo, N. A. Hill, A. Smythe, S. B. Chin, A. G. Johnson and N. Bird (2007b). Correlation of mechanical factors and gallbladder pain. Computational and Mathematical Methods in Medicine.

Li, W. G., X. Y. Luo, S. B. Chin, N. A. Hill, A. G. Johnson and N. C. Bird (2008). Non-Newtonian bile flow in elastic cystic duct: One- and threedimensional modeling. Annals of Biomedical Engineering 36(11), 1893-1908.

Luo, X., W. Li, N. Bird, S. B. Chin, N. A. Hill and A. G. Johnson (2007). On the mechanical behavior of the human biliary system. World Journal of Gastroenterology 13(9), 1384-1392.

Lygidakis, N. J. and W. H. Brummelkamp (1985). The significance of intrabiliary pressure in acute cholangitis. Surgery Gynecology and Obstetrics 161(5), 465-469.

Mark Feldman \& Lawrence S. Friedman \& Lawrence J. Brandt (2015). Sleisenger and Fordtran's Gastrointestinal and Liver Disease- 2 Volume Set, 10th Edition. Published by: SAUNDERS ELSEVIER- Philadelphia, United States.

Ooi, R. C., X. Y. Luo, S. B. Chin, A. G. Johnson and N. C. Bird(2004). The flow of bile in the human cystic duct. Journal of Biomechanics 37(12), 1913-1922.

Raptopoulos, V., T. M. Fabian, W. Silva, C. J. D'Orsi, A. Karellas, C. C. Compton, F.J. Krolikowski and E. H. Smith (1985). The effect of time and cholecystectomy on experimental biliary tree dilatation: A multi-imaging evaluation. Investigative Radiology 20(3), 276286.

Reinhart, W. H., G. Näf and B. Werth (2010). Viscosity of human bile sampled from the common bile duct. Clinical Hemorheology and Microcirculation 44(3), 177-182.

Reyes, H. and F. Kern (1979). Effect of Pregnancy on Bile Flow and Biliary Lipids in the Hamster. Gastroenterology 76(1), 144-150.

Rosen, J., J. D. Brown, S. De, M. Sinanan and B. Hannaford (2008). Biomechanical properties of abdominal organs in vivo and postmortem under compression loads. Journal of Biomechanical Engineering 130(2), 021020-1 021020-17.

Saxena, R. and N. Theise (2004). Canals of Hearing: Recent Insights and Current Knowledge. Seminars in Liver Disease 24(1), 43-48.

Schulte, S. J., R. L. Baron, S. A. Teefey, A.
Rohrmann, P. C. Freeny, W. P. Shuman and M. A. Foster (1990). CT of the extrahepatic bile ducts: Wall thickness and contrast enhancement in normal and abnormal ducts. American Journal of Roentgenology 154(1), 79-85.

Shawker, T. H., B. L. Jones and M. E. Girton (1981). Distal common bile duct obstruction: An experimental study in monkeys. Journal of Clinical Ultrasound 9(2), 77-82.

Sherratt, M. J. (2009). Tissue elasticity and the ageing elastic fibre. In Age 31(4), 305-325.

Slater, T. F. and V. B. Delaney (1971). The effects of various drugs and toxic agents on bile flow rate and composition in the rat. Toxicology and Applied Pharmacology 20(2), 157-174.

Sparks, J. L., N. A. Vavalle, K. E. Kasting, B. Long, M. L. Tanaka, P. A. Sanger, Karen Schnell and T. A. Conner-Kerr (2015). Use of silicone materials to simulate tissue biomechanics as related to deep tissue injury. Advances in Skin and Wound Care 28(2), 59-68.

St. George, C. M., J. C. Russell and E. A. Shaffer (1994). Effects of obesity on bile formation and biliary lipid secretion in the genetically obese JCR: LA- corpulent rat. Hepatology 20(6), 1541-1547.

Strasberg, S. M., R. N. Redinger, D. M. Small and R. H. Egdahl (1982). The effect of elevated biliary tract pressure on biliary lipid metabolism and bile flow in nonhuman primates. The Journal of Laboratory and Clinical Medicine 99(3), 342353.

Tanaka, M., S. Ikeda, and F. Nakayama (1983). Continuous measurement of common bile duct pressure with an indwelling microtransducer catheter introduced by duodenoscopy: new diagnostic aid for postcholecystectomy dyskinesia - a preliminary report. Gastrointestinal Endoscopy 29(2), 83-88.

Tomizawa, M., F. Shinozaki, R. Hasegawa, Y. Shirai, Y. Motoyoshi, T. Sugiyama, S. Yamamoto and N. Ishige (2017). Comparison of acute cholangitis with or without common bile duct dilatation. Experimental and Therapeutic Medicine 13(6), 3497-3502.

Vos, T., C. Allen, M. Arora, R. M. Barber, A. Brown, A. Carter, ... L. J.Zuhlke, (2016). Global, regional, and national incidence, prevalence, and years lived with disability for 310 diseases and injuries, 1990-2015: a systematic analysis for the Global Burden of Disease Study 2015. The Lancet 388(10053), 1545-1602.

Williams, R. D., J. C. Fish and D. D. Williams (1967). The Significance of Biliary Pressure. Archives of Surgery 95(3), 374-379.

Yamada, T., D. H. Alpers, A. N. Kalloo, N. Kaplowitz, C. Owyang and D. W. Powell(2009). Textbook of Gastroenterology, Fifth Edition. In Textbook of Gastroenterology, Fifth Edition Wiley-Blackwell: New Jersey, United States. 